\title{
Smart Care Beds for Elderly Patients with Impaired Mobility
}

\author{
Youn-Sik Hong (D) \\ Department of Computer Science and Engineering, Incheon National University, Incheon 406-772, Republic of Korea \\ Correspondence should be addressed to Youn-Sik Hong; yshong@inu.ac.kr
}

Received 19 February 2018; Accepted 29 July 2018; Published 12 August 2018

Academic Editor: Seyed M. Buhari

Copyright (C) 2018 Youn-Sik Hong. This is an open access article distributed under the Creative Commons Attribution License, which permits unrestricted use, distribution, and reproduction in any medium, provided the original work is properly cited.

\begin{abstract}
Most accidents occurring at medical institutions treating elderly patients with mobility impairment are bedsores and fall accidents. One of the reasons for this high rate of accidents is the lack of nursing personnel. In order to aid caregivers in nursing elderly patients who are not able to move about freely, in this paper, we propose a design and implementation of a smart bed. In this bed, several pressure sensors are deployed underneath the mattress cover to consider both peoples' standard physical characteristics and the specific body parts where bedsores commonly occur. To manage the pressure ulcer area and to prevent falls, the body area is divided into three vertical areas and three horizontal areas. Each microcontroller unit manages pressure-sensing information in one of the body regions divided horizontally. In this study, a real-time pressure-sensing algorithm is presented that is capable of deciding on the possibilities of bedsores and falling accidents by considering both the intensity and the duration of pressure of specific body parts. Our experimental results demonstrate that a prototype smart bed works well for several human models of various heights and weights.
\end{abstract}

\section{Introduction}

In recent years, South Korea has faced the challenge of the rapidly aging society. Therefore, the problem of the elderly patients' care becomes crucial. In addition, an increasing number of specialized medical institutions for the elderly and senior care centers (called sanatoria) have been established. However, the rapid growth of the industry related to the elderly care and the establishment of relevant institutions encounters many problems, including, among others, the lack of appropriate administration.

If an elderly individual is in the same position for too long, whether in bed or a wheelchair, the friction between the skin and the surface may stop the blood flow, causing the pressure area to receive less oxygen and thus causing the cells to die in that area. This is how bedsores will develop [1]. Pressure ulcers in elderly individuals can cause significant morbidity and mortality and are a major economic burden to the healthcare system [2].

Recent surveys [3] have estimated a huge expenditure on direct medical cost for domestic accidents (in the USA, more than US $\$ 19$ billion for nonfatal falls in subjects $>65$ years of age; in the UK, US $\$ 1.9$ billion for emergency services and hospitalization, while an Australian study reported a national healthcare cost for elderly falls of US $\$ 66.1$ million). Interestingly, two-thirds of the overall medical costs are due to falls requiring hospitalization [3]. Falls are by far the leading cause of injuries among the elderly [4]. One out of every 3 seniors aged 65 and older experiences a fall each year. Falls are the leading cause of both nonfatal and fatal injuries among seniors, and 20 to 30 percent suffer moderate to severe injuries that can cause disability or increase the risk of death. From a healthcare perspective, elderly falls are a major clinical issue in terms of frequency, disability, institutionalization, and overall mortality, with an outgrowing socioeconomic burden [5].

The occurrences of bedsores and fall accidents among the elderly patients are typical problems, accounting for over $80 \%$ in such medical institutions [6]. The Ministry of Health and Welfare of Korea has introduced operational regulations for medical institutions and, using these regulations, the latter measure the risk of falls and pressure ulcers. Nevertheless, the number of occurrences of bedsores and fall accidents is still growing. The main reason behind this tendency is the lack of trained personnel. 
Internet of Things (IoT) revolution is redesigning modern healthcare with promising technological, economic, and social prospects [7]. Enabled by the global connectivity of the IoT, all the healthcare information (logistics, diagnosis, therapy, recovery, medication, management, finance, and even daily activity) can be collected, managed, and utilized more efficiently [8]. Among the panoply of applications enabled by the IoT, smart and connected healthcare is a particularly important one. Networked sensors, either worn on the body or embedded in our living environments, make possible the gathering of rich information indicative of physical and mental health [9]. By the current technology status, wearable health management systems [10] provide low-cost solutions for ubiquitous, all-day, unobtrusive personal health monitoring and are expected to enable early detection and better treatment of various medical conditions as well as disease prevention and better understanding and self-management of chronic diseases. In addition, RFID technology is to provide part of the IoT physical layer for the personal healthcare in smart environments through low-cost, energy-autonomous, and disposable sensors [11].

In this paper, we have implemented an IoT-based smart bed system designed to aid caregivers to nurse elderly patients with mobility impairments. The primary goal of the smart bed system is to prevent bedsores and/or falling accidents from the bed.

For that purpose, two types of pressure sensors (strip and square type) were used to sense pressures in the specific body positions depending on sensing purposes. We used sensors regarding both the average body size by age (with reference to the Korea Agency for Technology and Standards (KATS)) and the frequent location of bedsores. Besides, the entire sensing area of a patient's body was divided into three subareas and each of them was monitored by a distinct module operating independently for fault tolerant purposes. In addition, we also introduced the pressure-sensing algorithm that accurately detects the pressure intensity and the duration of the pressure. A short version of this paper has been published elsewhere [12].

The remainder of this paper is organized as follows. Section 2 summarizes related work. In Section 3, we describe the overall architecture and detailed operations of a smart bed system. In Section 4, the experimental results used to validate the proposed method are presented. Finally, conclusions are drawn in Section 5.

\section{Related Works}

The work in [13] proposed a computer vision-based fall detection system for monitoring an elderly person in a home care application. It suffers from occlusion since subjects can sometimes be behind a sofa or furniture while being monitored [14]. Gasparrini et al. [15] developed a fall detection system using a Microsoft Kinect depth sensor. It uses two sensors, an infrared projector and an infrared sensor, to generate a $3 \mathrm{D}$ map of the scene [15]. The use of a 3D map allows differentiating between obstructions and the subject. It can detect a fall-down accident through the analysis of a patient's gestures. The fall-down prevention device [16] is similar to the approach of Gasparrini et al. [15]. The drawback of the above approaches is the increase in computing complexity and cost. In addition, these methods do not prevent the occurrence of bedsores.

Providing accurate and opportune information on people's activities and behavior is one of the most important tasks in pervasive computing. Lara et al. [17] survey the state of the art in human activity recognition based on wearable sensors [17]. Ozcan et al. [18] present a fall detection system using wearable cameras. Since the camera is worn by the subject, monitoring is not limited to confined areas and extends wherever the subject may go including indoors and outdoors. When a fall occurs, edge orientations in a frame vary drastically and extremely fast, as a result of this subsequent frames get blurred [14].

Another work [19] is a system of unobtrusively recognizing actions and promoting physical activities and a smart mat used for the same purpose. A mat sensor is constituted by a FSR (force sensing resistor) sensor, a load cell, and a piezoelectric sensor. In addition, an ultrasonic sensor, an infrared sensor, and a thermal sensor are attached outside to monitor the behavior information of a patient lying on the mat. However, since the mats require the use of numerous sensors, their application in senior care centers is too complex and expensive. Furthermore, this patent may be more problematic to apply due to its complicated structure. Among other reasons, power consumption is increased, and the mobility of the bed is not free, as the external power source must be connected. Maintenance is also difficult. However, this patent is advantageous to grasp the behavior of a person to be protected from various sensors, although there appear to be restrictions in terms of commercialization.

BodiTrak Smart Bed [20] uses its own elastic and breathable pressure-sensing fabric. As one lies on it, the system continuously monitors one's body position and pressure distribution and automatically adjusts the bed's air support for maximum comfort. It is a system that keeps pressure distribution balanced for comfortable sleep. There is similarity with the method to be proposed in this paper in terms of utilizing the pressure distribution, but both the purpose of application and the application technique are quite different.

Adaptive sleep thinking system [21] has five independent zones adjusting to relieve pressure and improve sleep quality. This system is functionally similar to [20]. The difference between these two systems is that the system [20] divides the pressure generating part of the body into five zones and independently controls each of the five zones.

MAP system [22] involves a special electronic sheet placed over a mattress that has thousands of sensors that detect the pressure distribution of the patient's body over the bed. It is very similar to our proposed method. However, our proposed system uses 1/20 times fewer sensors than MAP. In addition, depending on the upper body and lower body, different types of sensors are deployed efficiently considering the incidence of bedsores. Notice that the incidences of bedsores are relatively low in the areas with frequent movements such as legs. 


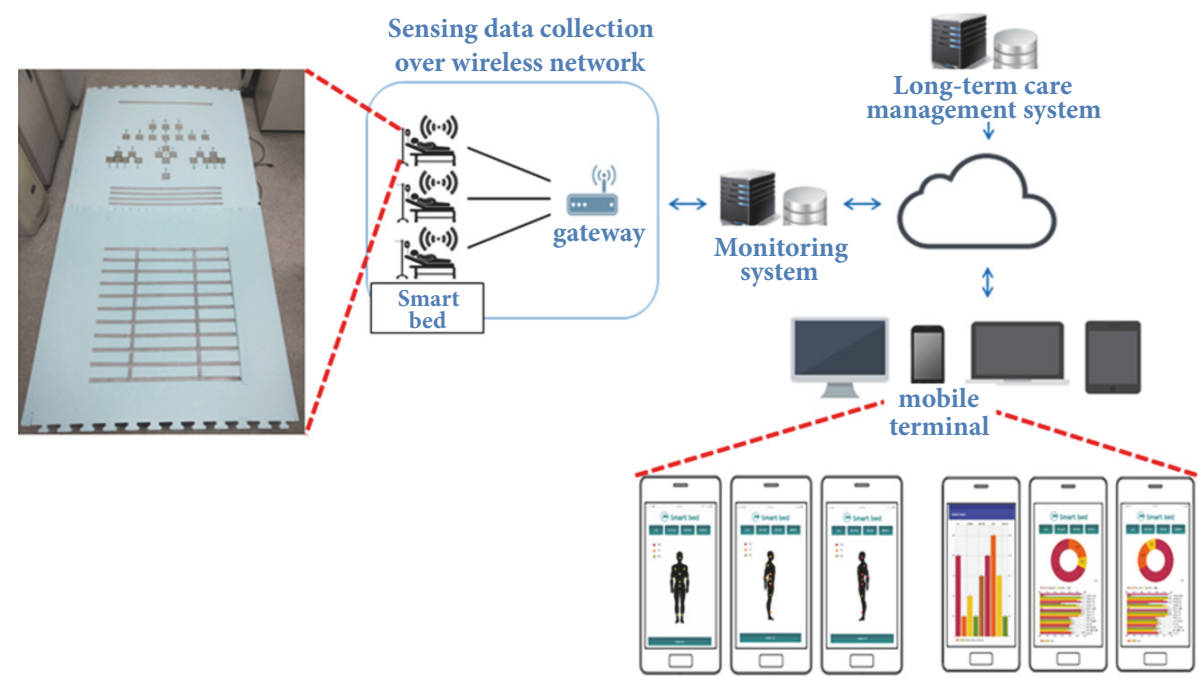

FIgURE 1: The H/W diagram of a smart bed system.

\section{The Smart Bed System}

3.1. System Overview. The primary purpose of the proposed smart bed system is to assist caregivers in nursing elderly patients who are not able to move about freely. With a smart bed, several pressure sensors installed under the mattress cover measure pressure intensity due to the weight of the patient lying on the bed. A smart bed can provide the information about pressure intensity of the points or areas of the patient's body. With this information, we know the patient's posture, that is, the supine position or lateral position, and the duration of pressure in a particular body position. This knowledge can help caregivers in terms of preventing the appearance of bedsores and/or falling accidents from the bed.

One of the most important functions of the smart bed system is that it can easily find the specific points of the patient. Based on the average body size by age (with reference to the KATS), a smart bed system is able to recognize the known points where bedsores frequently occur, that is, occiput, scapula, superfine projections, elbow, iliac crest, sacrum, pubic bone, the Achilles tendon, heel, etc. [23]. Figure 1 shows the block diagram of the smart bed system.

3.2. Design of a Smart Bed System. Our smart bed meets the standard requirements of $1,895 \mathrm{~cm} \times 850 \mathrm{~cm}$, which are the dimensions of a bed for medical purposes. The entire body area of a patient is divided into three distinct sections (see Figure 2). This classification has been performed based on pressure intensity and the location of the frequent occurrence of bedsores. The points marked as dots in each section have a similar pressure intensity and they are close to the known position of bedsores.

The first section corresponds to the upper body. It consists of the head, with the three dots, namely, occiput, scapula, and superfine projections, respectively. The second section consists of arms and buttocks, with the following four dots: elbow, iliac crest, sacrum, and pubic bone. The third section consists of legs, with two dots for calf and heel (see Figure 2).

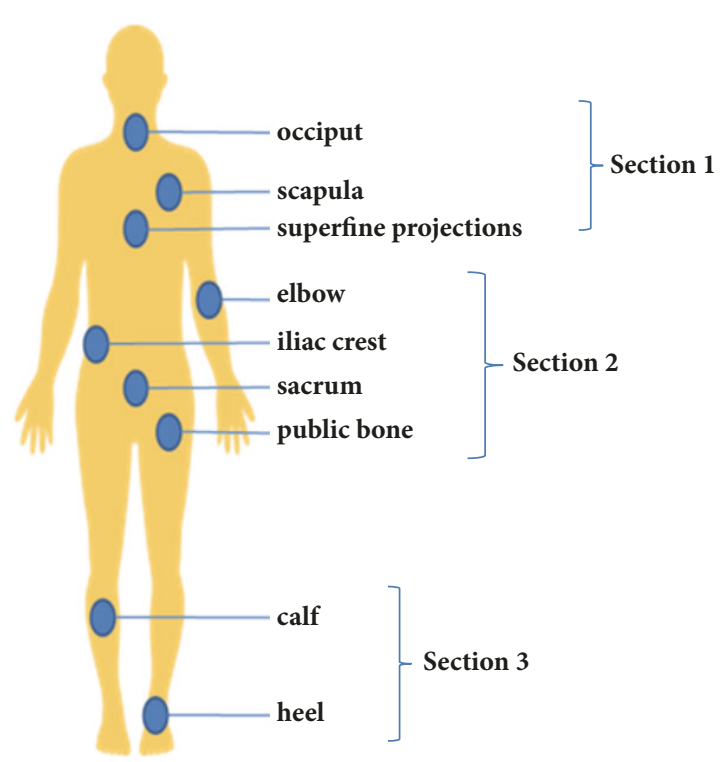

FIgURE 2: The three distinct sections and the positions of the frequent occurrence of bedsore.

In order to collect the data on pressure intensity sent from the sensors, a microcontroller unit (MCU) is allocated in each section. It has been implemented with Arduino MEGA2560. Since each MCU operates independently, the smart bed system performs its own work, even though one or two MCUs have some troubles or stopped accidentally.

As shown in Figure 1, the gateway to the monitoring system is implemented with the Raspberry PI board. It has an ATmega644 CPU and operates on the Linux operating system. The gateway communicates with three distinct MCUs via the Master-Slave mode [24]. Each MCU periodically transmits the pressure data to the gateway through wireless communication using Wireless LAN (WLAN). The gateway 

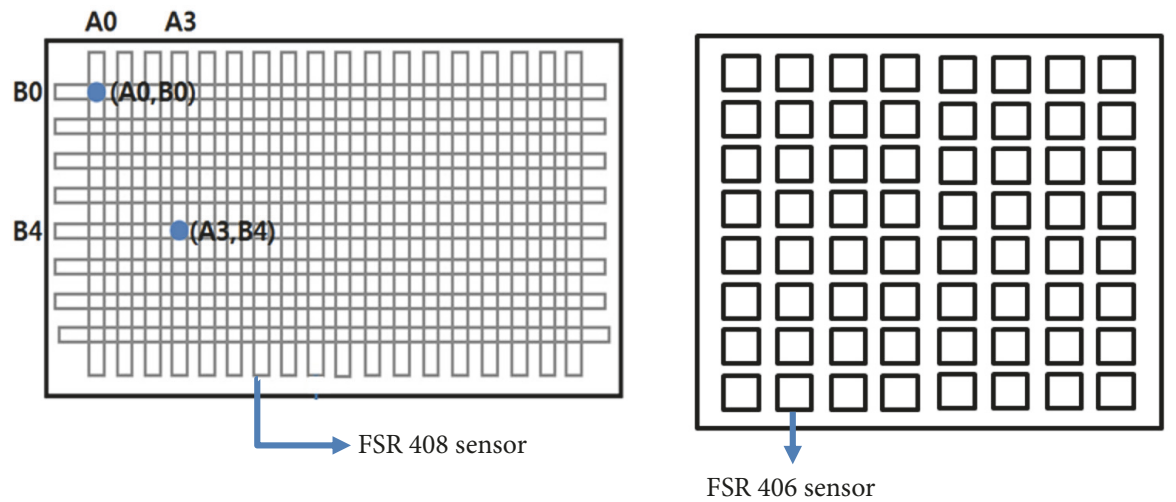

FIGURE 3: Deployment of FSR-406 and FSR-408 sensors.

converts these raw data into the corresponding formatted data and then stores them in the local database.

These data will be sent to the long-term care management system (LCMS) and then be stored in a permanent database. Finally, they will be used as persistent data for the LCMS. In the case of a high possibility of a bedsore attack or a falling accident from a bed, an alarm message will be sent to the caregiver's smart device immediately via the Google FCM (Firebase Cloud Messaging) server.

3.3. Deployment of Two Types of Sensors. The pressure sensor needs to be arranged according to the average body size of each age group. In particular, if the pressure of a specific body part is to be measured, the body part dimensions for each age group should be reflected in the sensor placement process. We have deployed sensors regarding both the average body size by age, as based on the KATS, and the position of the frequent occurrence of bedsores. Doing so will make it possible to detect pressure of body positions with various body dimensions.

Our design goal for sensor deployment is to use the minimum number of sensors to detect pressure of all possible body positions. Depending on the body part, there are areas that require precise measurement of pressure and others that do not. For example, instead of measuring the pressure of a specific part of the head and the lower body, it is only necessary to measure whether or not the pressure is generated. On the other hand, the upper body needs to measure the pressure closely around a specific position where the pressure ulcer frequently occurs. In this situation, we will use two kinds of sensors: FSR-408 and FSR-406 [25]. These sensors are chosen in terms of cost effectiveness and ease of implementation. According to the data sheet [25], this force sensitivity is optimized for use in human touch control of electronic devices such as automotive electronics, medical systems, and industrial and robotics applications. The FSR408 sensor (strip-type) is used to detect whether a pressure has been generated. On the other hand, the FSR-406 sensor (square type) is used to precisely measure the pressure of a specific area. The dimensions of FSR-408 and FSR-406 are $610 \mathrm{~mm} \times 5 \mathrm{~mm}$ and $38 \mathrm{~mm} \times 38 \mathrm{~mm}$, respectively. The pressure range for the above two sensors is $0.1 \sim 10.2^{2} \mathrm{~N}$ (Newton).
Both FSR-406 and FSR-408 are two-wire devices with a resistance that depends on the applied force. The measuring resistor output voltage increases with the increasing force.

The FSR-408 pressure sensor can detect the pressure of a wide area with relatively smaller numbers. It knows only the strength of the pressure, whereas it does not know the exact position of the issued pressure. To use FSR-408 sensors, they are deployed in a grid pattern (see the left part of Figure 3).

Note that, as shown in Figure 4, they are used to detect pressures of patient head, legs, and hips. The vertical spacing between the sensors for detecting pressure of hips is 20 $30 \mathrm{~mm}$, whereas the vertical spacing for legs is $50 \mathrm{~mm}$. To expect the position of issued pressure, two FSR sensors are necessary. For example, assume that two sensors, A0 and B0, in the left part of Figure 3 detect pressures simultaneously. Then, the intersection point (A0, B0) marked as a dot will be expected as the position of pressure issued. Another intersection point (A3, B4) will be the same as in the previous example.

To detect pressure of the upper body, FSR-406 sensors are deployed in a dot pattern (see the right part of Figure 3). FSR-406 sensor does know the exact position of the issued pressure. They have been deployed by $190 \mathrm{~mm}$ distance away from the FSR-408 sensors deployed in the back of the patient head (called occipital area sensors) to detect the pressure of the shoulder portion. Note that the exact dimensions are determined by using the Korean standard body dimensions from KATS. For example, this distance is measured for Koreans to amount to $190.223 \mathrm{~mm} \sim 220.44 \mathrm{~mm}$. FSR-406 sensors are also deployed to detect pressure of both superfine projections (spine) and elbow positions.

For the actual implementation (see the left part of Figure 5), a total of 45 sensors are used: 18 FSR-408 sensors and 27 FSR-406 sensors. The FSR-406 sensor and the FRS408 sensor are priced at $\$ 10$ and $\$ 25$, respectively. The cost of implementing the entire system is only one-fifth of the cost of competing systems. With this implementation, 22 positions of the frequent occurrence of bedsore can be detected: 10 positions for the supine posture and 12 positions for the lateral posture. Such implementation is designed for elderly patients whose height is within the range of $150 \sim 180 \mathrm{~cm}$. 

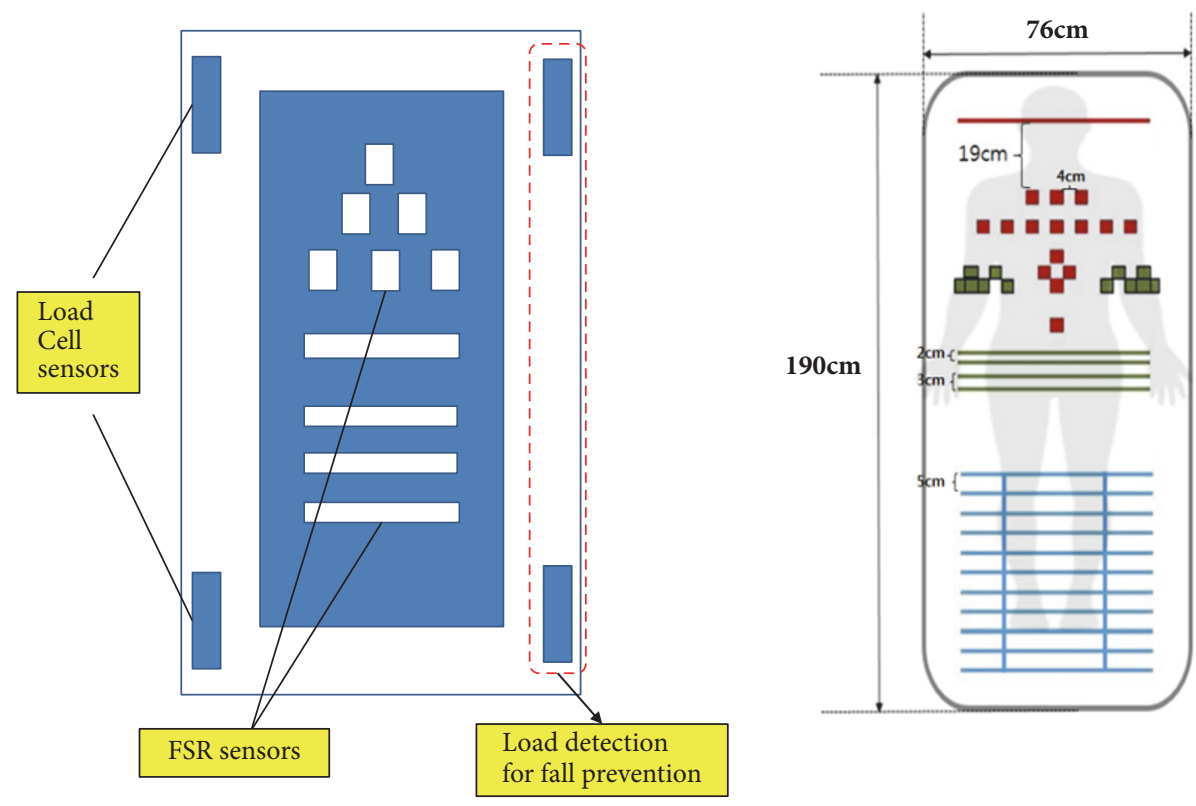

FIgURE 4: The placements of load-cell sensors and FSR sensors (left) and of the two types of sensors using a grid pattern and a dot pattern (right).
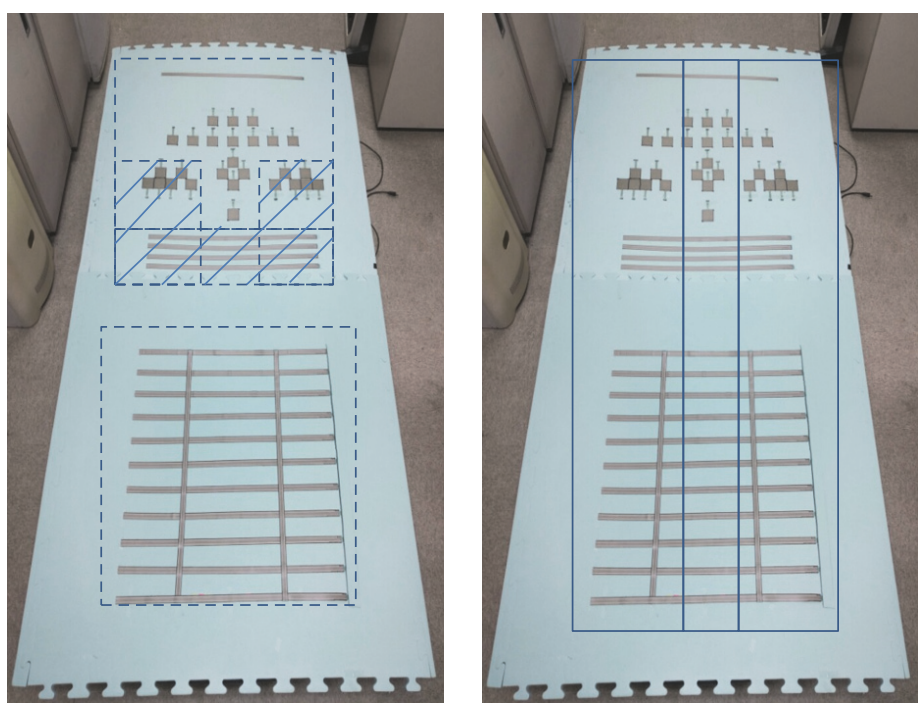

FIGURE 5: The 3 horizontal subsets of sensors (left) and the 3 vertical subsets of sensors (right).

3.4. Sensing Algorithm for Fall Risk Assessment and Pressure Ulcer Occurrence Warning. As shown in the left part of Figure 4, four load cells for detecting the load are placed on the edge of the bed. They can determine whether the patient is in bed and measure the patient's weight if the patient is lying down properly. In Figure 4, the dot-type (FSR-406) sensor and the strip-type (FSR-408) sensor arrangement are shown on the right. Note that the dimensions of the main parts are also shown.

The set of FSR sensors can be divided into three groups according to the sensing purpose, as shown in Figure 5, either vertically or horizontally. The left side of Figure 5 divides the FSR sensors horizontally into three subsets to measure the pressure of the major body parts. Three MCUs process the pressure information of the sensors of each subset divided in the horizontal direction. Each sensor subset in the horizontal direction is used to monitor whether the pressure at the frequent occurrence of bedsore has been maintained for a certain period of time.

As shown on the right part of Figure 5, the FSR sensors are arranged vertically in three groups: left, center, and right. Depending on how many sensors of one subset, as compared to the other two subsets, sense pressure, we can expect the patient's posture in the bed and thus generate the warning message against a falling accident. If over $80 \%$ of the sensors of one of the groups except for the sensors of the central group 


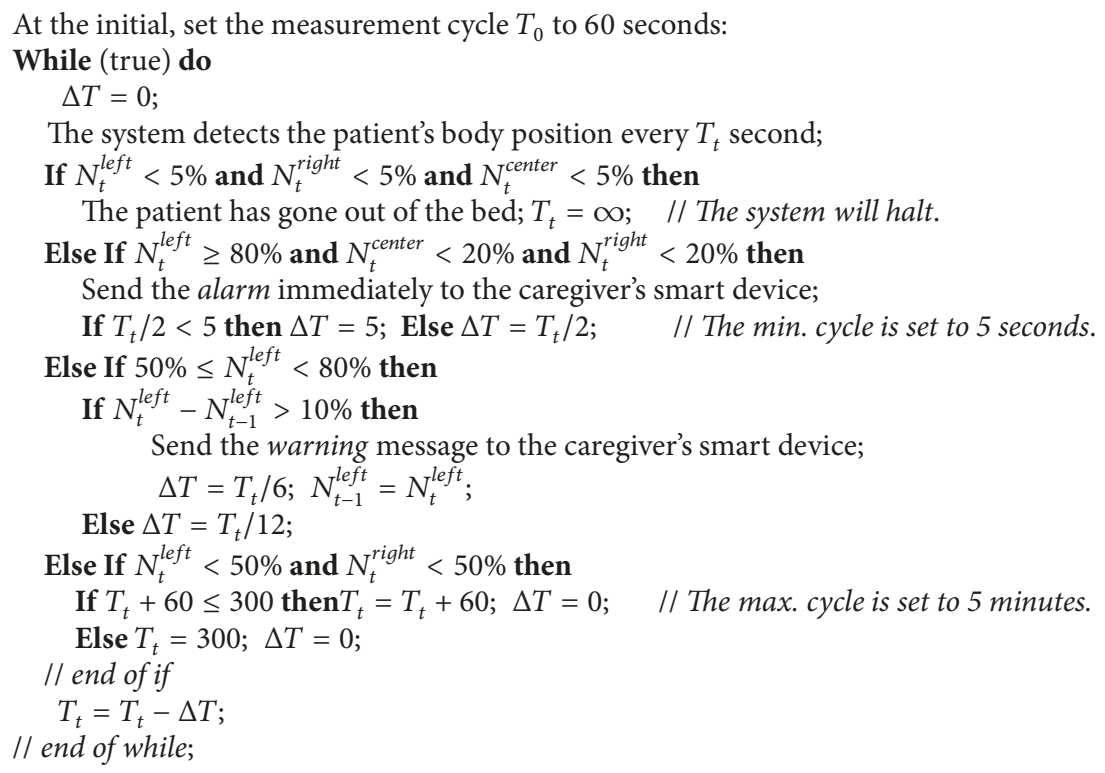

Algorithm 1: The sensing algorithm for fall risk assessment.

sense the pressure and, at the same time, less than $20 \%$ of the sensors of the remaining group sense the pressure, it can be decided to be one of the following two case: either the patient lying on the bed is in danger of falling or s/he is coming down from the bed. In any case, the caregiver will be immediately notified that the patient needs protection.

The system detects the patient's position every 1 minute. However, as the possibility of fall accident increases, the measurement period will be shortened to 30 seconds or less. In Algorithm 1, we present a sensing algorithm for the fall risk assessment.

Let $N_{t}^{\text {left }}$ be the percentage of sensors of the left vertical group which sense pressure at time $t$. Similarly $N_{t}^{r i g h t}$ and $N_{t}^{\text {center }}$ are the percentage of sensors of the right vertical group which sense pressure and the percentage of sensors of the center vertical group which sense pressure at time $t$, respectively. Note that, for simplicity, Algorithm 1 describes only the detection of fall accident that occurs in the left end of the bed. In this case we assume that the safety guard on the right side of the bed is raised so that the patient cannot fall down to the right.

As the ratio of the pressure sensed by one group of sensor increases, the measurement cycle $T$ starts to shorten. If the percentage of sensors of the left group sensing pressure increases by over $10 \%$ after $\mathrm{T}$ interval, the next cycle $\mathrm{T}$ will be decreased by half of the current cycle.

After a fall risk warning, if the pressure is no longer measured anywhere on the bed, it is decided that the patient is no longer in bed. It is judged that a fall has occurred or the patient has gone out of bed and moved to another place.

To prevent ulcer occurrence, perform a field test to determine the patient's posture (supine, repose, left position, right position, half position, etc.) according to the pressure pattern applied to the bed. In addition, when a patient lies in the bed, the height of a patient is calculated using the distance between the back head sensor and the farthest distance sensor based on the sensor located at the heels of the patient. In this way, the body part with frequent pressure ulcers is sought. Assume that the background data (gender, age, height, and weight) for the patient are given. A sensing algorithm for pressure ulcer occurrence warning is shown in Figure 6.

Finally, the system implements the following features:

(1) Visualizing and displaying the duration of pressing for each body part.

(2) The graph shows the current status and daily and weekly cumulative status of the patient.

(3) If the same site is continuously pressed for longer than 30 minutes, a warning message will be generated.

\section{Experiments}

4.1. The Detection Rate of the Pressures of the FSR Sensors. In this experiment, we examine whether the FSR sensors accurately detect pressure. This is because the smart bed system can accurately determine the patient's posture according to whether the pressure is properly detected. If the value sensed by a FSR sensor is below the reference value (i.e., threshold), it cannot be used for a determination of lying posture. In general, a FSR sensor can detect the value above the threshold if a patient lying in the bed maintains a constant posture for a certain period.

The pressure range of a FSR sensor is $0 \sim 1,023$. The threshold used in this experiment is 150 , where it was determined empirically. In addition, the detection rate of the pressures of the FSR sensors has been obtained when the patient was lying in the same position for more than one minute (see Figure 7). The values shown in Table 1 are the 
Determine the patient's posture: supine or lateral (left, right) position ;

7

Find the two sensors which sense the pressure: the back head sensor $S_{\text {heel }}$ and the heel sensor, $S_{\text {head }}$, where these two sensors are the farthest distance from each other;

Calculate the height of the patient using the distance between $S_{\text {head }}$ and $S_{\text {heel }}$;

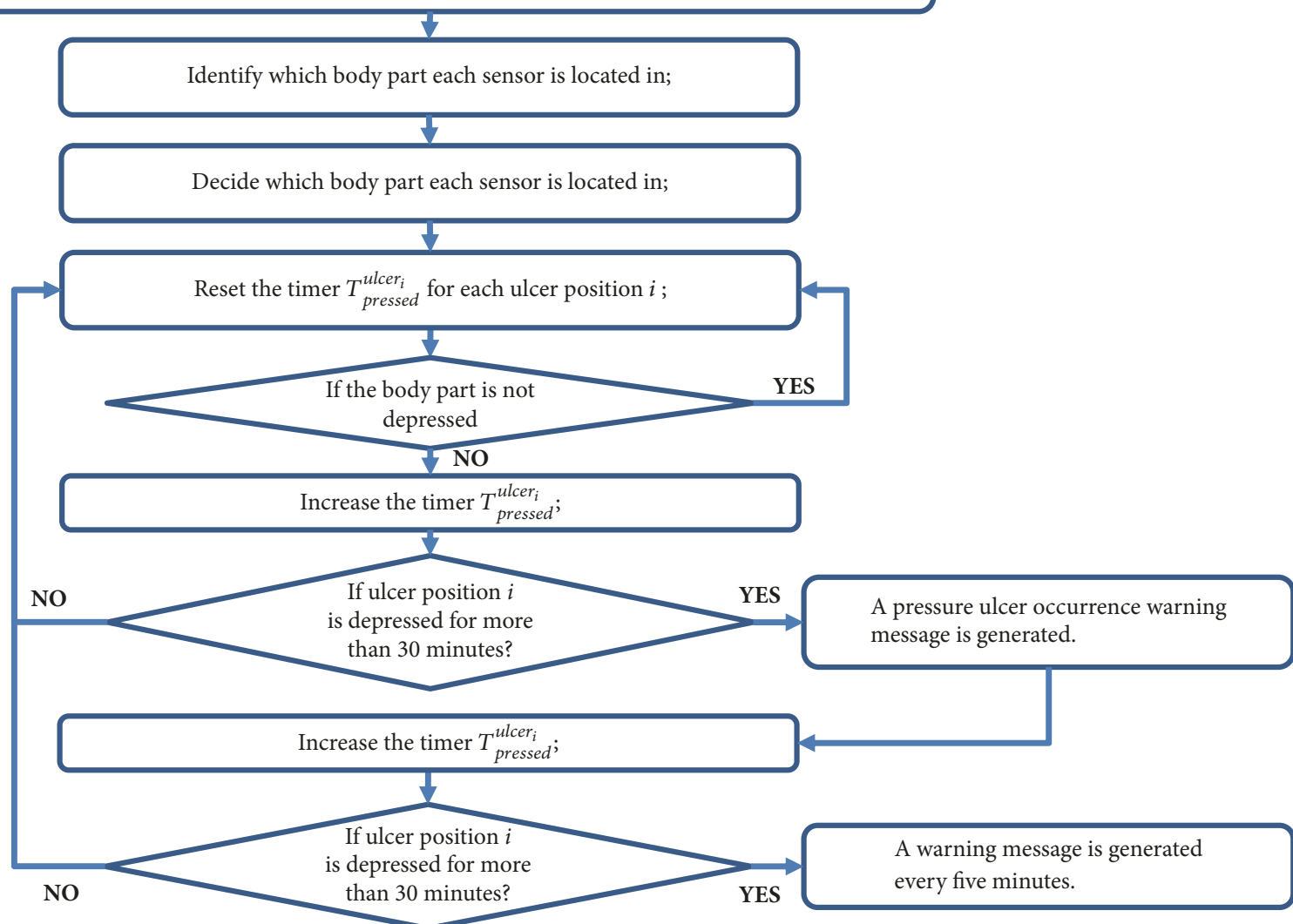

FIGURE 6: The sensing algorithm for pressure ulcer occurrence warning.

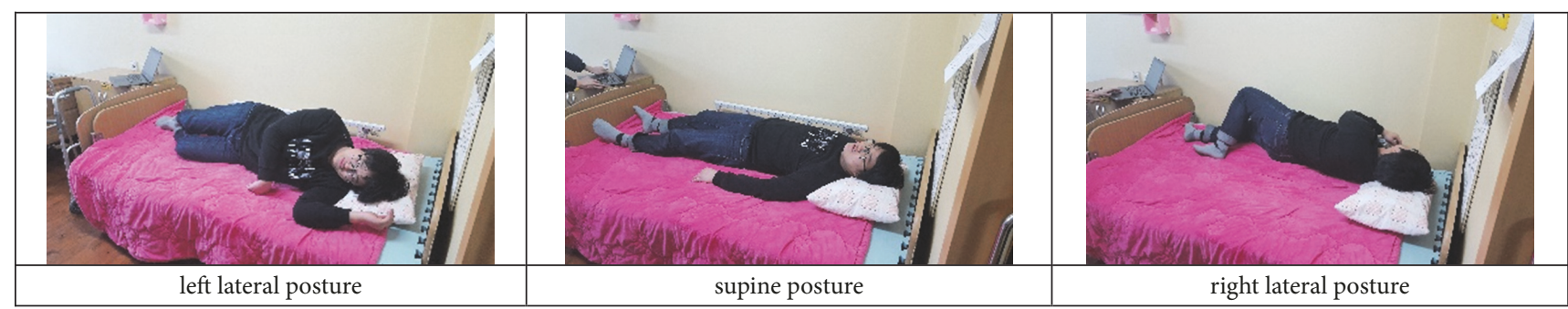

FIGURE 7: Measurement of the detection rate of the pressures with respect to the lying posture.

number of FSR sensors whose values were measured above the threshold. The measurements were repeated 20 times for each posture. The detection rate when lying down in normal posture (a.k.a. supine posture) was higher than the left or right lateral posture.
4.2. The Recognition Rate of the Lying Posture. This is an experiment to evaluate the performance of the algorithm to determine what posture the patient is lying on using the pressure values sensed by the FSR sensors. Notice that the smart bed system can determine the posture when the patient 
TABLE 1: The detection rate with respect to the lying posture.

\begin{tabular}{lcccc}
\hline lying posture & $\begin{array}{c}\text { total no } \\
\text { of sensors }\end{array}$ & $\begin{array}{c}\text { min. no } \\
\text { of sensors }\end{array}$ & $\begin{array}{c}\text { max. no } \\
\text { of sensors }\end{array}$ & $\begin{array}{c}\text { average no } \\
\text { of sensors }\end{array}$ \\
\hline right lateral & 26 & 23 & 26 & 24.55 \\
\hline left lateral & 26 & 23 & 26 & 24.49 \\
\hline supine & 27 & 24 & 27 & 25.48 \\
\hline
\end{tabular}

TABLE 2: The recognition rate with respect to the threshold.

\begin{tabular}{lccc}
\hline threshold & no of tests & $\begin{array}{c}\text { successful } \\
\text { recognition }\end{array}$ & recognition rate (\%) \\
\hline 50 & 112 & 85 & 75.9 \\
\hline 150 & 128 & 104 & 81.3 \\
\hline 300 & 134 & 117 & 87.3 \\
\hline
\end{tabular}

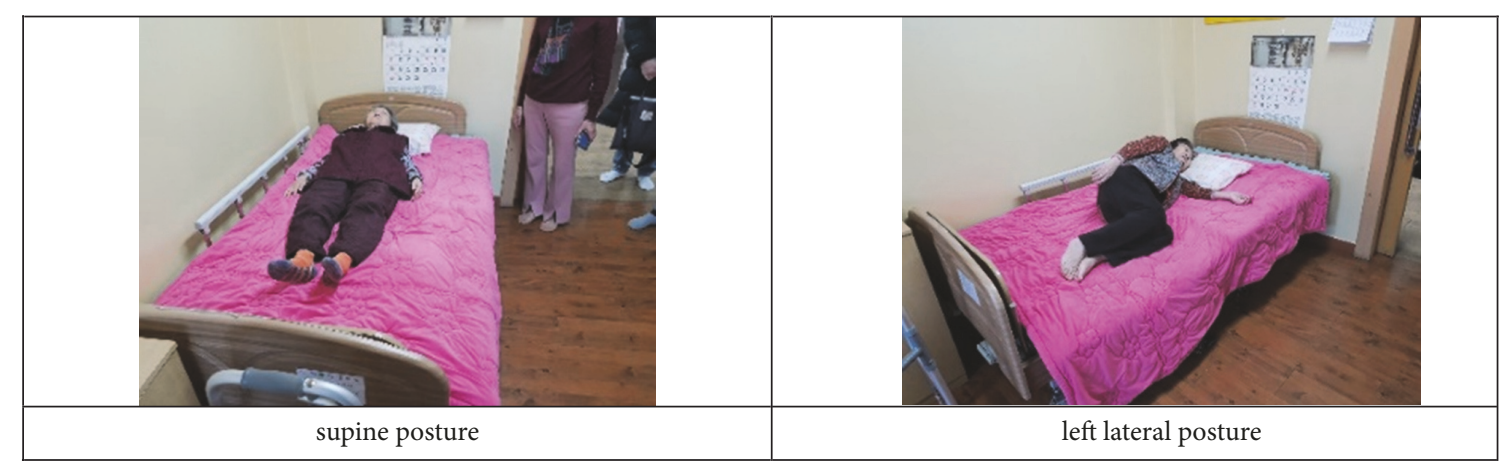

FIGURE 8: Experiments for recognizing a patient's posture based on the pressures measured.

is lying in the same position for more than one minute. This delay considers all the delays due to the processing delay, the propagation delay over wireless, and the database processing delay. Experiments were conducted on elderly people in the elderly care facilities (see Figure 8).

As shown in Table 2, for this experiment, three threshold values were used: 50,150, and 300. As the threshold value becomes greater, the rate of discriminating correct posture increases. Head, shoulder, and hip are the body parts where their pressure values are higher than those of the other parts. The larger the threshold value, the more accurate the main body parts can be measured. If head, shoulder, and hip are recognized as straight lines based on the pressure value, the patient can be judged to be lying in the supine posture. To make such a decision, it is necessary to accurately measure the pressure values corresponding to the body part.

One of the reasons for not recognizing the patient's posture is that when lying down the bed the behavior patterns and the lying postures are different for each patient. This type of the recognition error due to different behavior patterns for each patient can be effectively reduced through data accumulation and analysis. Another reason is that the patient's body size varies. When installing the system, we can reduce the rate of recognition error by adjusting the parameters to match the patient's body size. The values in
Table 2 are the results of the experiments without these adjustments.

4.3. The Communication Delay over Wireless. The pressure values sensed by the FSR sensors are transmitted to the Arduino board (Arduino Mega-2560) as explained earlier in Section 3.2. It can communicate with the Raspberry PI board via serial communication at 9,600 bps baud rate. Then it periodically transmits the stored pressure values to the Raspberry PI. Finally, the Raspberry PI wirelessly transmits them to the remote server through IEEE 802.11n WLAN. Notice that the payload data size used in the experiment is 256 Kbytes.

Experiments assume a single-hop wireless transmission from the Raspberry PI to the server. We measured the time it takes for the values detected by all the FSR sensors to reach the server. This value is used as a basis for estimating the transmission time of an SMS message when an emergency such as a fall accident or a high occurrence of a pressure ulcer happens.

As summarized in Table 3 and also shown in Figure 9, the wireless transmission delay is less than $2.5 \mathrm{~ms}$. Based on this value, it is possible to estimate the minimum period required to store the pressures values in the remote database system. In other words, the minimum interval of the data transmission for the smart bed system can be obtained. Since 
TABLE 3: The statistics of the communication delay over WLAN.

\begin{tabular}{lc}
\hline No of tests & 142 \\
\hline average delay & $1.616[\mathrm{~ms}]$ \\
\hline minimum delay & $1.238[\mathrm{~ms}]$ \\
\hline maximum delay & $2.435[\mathrm{~ms}]$ \\
\hline
\end{tabular}

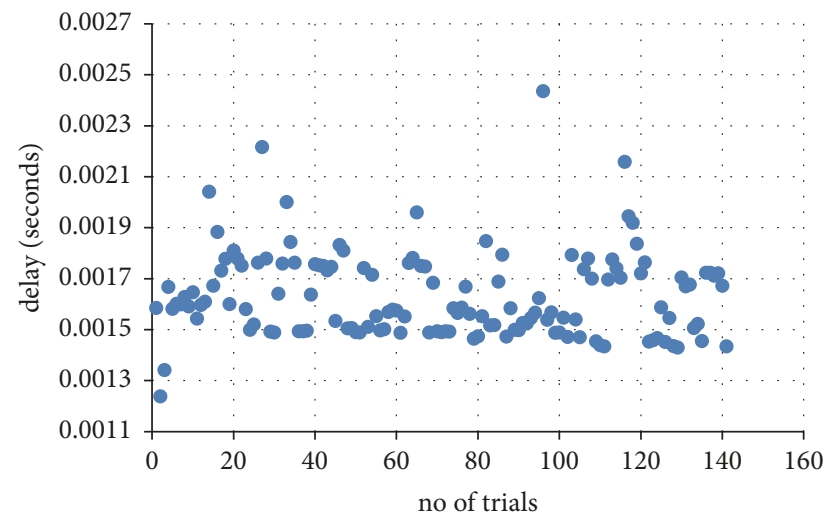

Figure 9: The distribution of the communication delays over WLAN.

the processing delay is less than $1 \mathrm{~ms}$, the minimum period becomes $3.5 \mathrm{~ms}$. Since these experiments were conducted in the laboratory where there is little traffic in WLAN, it may increase in more realistic environments.

However, it is not necessary to transmit the pressure values in a shorter period less than 1 minute unless there is a risk of fall accidents or occurrence of pressure ulcers. As the transmission period becomes shorter, the number of records inserted into the database increases rapidly. Therefore, it is necessary to adjust the transmission period appropriately. The transmission cycle seems to be appropriate in 3 to 5 minutes in a normal situation.

4.4. The Delay of Transmitting SMS to the Registered User. We used Google FCM, a cross platform messaging solution, to implement emergency text messaging. Performance was measured at maximum payload $4 \mathrm{~KB}$ including dummy data. The mobile application for users is implemented on the Android platform. The number of mobile terminals to be transferred at the same time was limited to one registered terminal to protect patient information. User authentication uses the sender ID, and the message lifetime is set to only one day. Normally, the message lifetime is set to 2,419,200 seconds (i.e., 28 days). We measured message transmission time and message reception time, respectively.

As shown in the left of Figure 10, we assumed that the high possibility of falling accidents occurs and then the smart bed system sends emergency text message to the registered caregiver. The average delay is 0.74 seconds and the maximum delay is 2.64 seconds, as summarized in Table 4 . This is because the variation of the delay is influenced by network traffic at the time of transmission and reception. In the performance evaluation, the measurement was made on one registered terminal, but the maximum delay was within 5
TABLE 4: The statistics of the delays of transmitting text message to the one registered user.

\begin{tabular}{lc}
\hline No of tests & 142 \\
\hline average delay & $0.737[\mathrm{~s}]$ \\
\hline minimum delay & $1.096[\mathrm{~s}]$ \\
\hline maximum delay & $2.643[\mathrm{~s}]$ \\
\hline
\end{tabular}

TABLE 5: The processing time with respect to the size of D-zone.

\begin{tabular}{lcc}
\hline type & no of sensors & processing time $[\mathrm{ms}]$ (avg.) \\
\hline min. D-zone & 10 & 0.115 \\
\hline medium D-zone & 17 & 0.268 \\
\hline max. D-zone & 24 & 0.412 \\
\hline
\end{tabular}

seconds even when the message was transmitted to two terminals at the same time.

4.5. Detection of Falling Accident. We applied the prototype smart bed to two groups of patients: elderly patients who can hardly move and elderly patients with dementia who can move a little. In the case of the former group, little change in the pressure sensor value over time was observed. However, pressure changes occurred frequently in the latter group of patients. Specifically, in this group, it took at least 2.7 minutes (on average) from the time when the left (or right) pressure ratio of the bed reached $80 \%$ to the time when the patient came down from the bed. The fastest alarm cycle was set to 5 seconds, indicating that the caregiver had sufficient time to recognize the alarm and then come back to the patient. Note that, in the experiment, the caregiver was assumed to be on the same floor as the patient. Besides, the caregiver was assumed to be within the 100-meter reach from the patient.

It is not easy to analyze the relationship between detection time and prevention of falls. This is because it is hard to find out what an elderly patient will do on one side of the bed. Therefore, the proposed method only determines whether the patient's position is located one side of the bed as shown in Figure 5. It focuses on informing a caregiver as soon as possible when the patient's position reaches a dangerous zone (called $D$-zone), such as a corner of the bed, so that she (he) can identify the situation.

Experiments were conducted to investigate the effect of the size of D-zone on detection time. This is because limiting the search space can be effective for reducing detection time to prevent fall if a patient moves to one side of the bed. For the purpose of the experiment, the 3 types of $\mathrm{D}$-zone are defined as shown in Figure 11. The minimum D-zone has only 10 sensors, while the maximum D-zone has 24 sensors.

Depending on the $\mathrm{D}$-zone size, the processing time may vary, summarized as Table 5 , but the total elapsed time heavily depends on the time it takes to send the text message to the caregiver.

$1 \mathrm{~ms}$ (processing cycle $)+2.435 \mathrm{~ms}$ (delay over wireless $)+$ 2.643 s (delay of transmitting $S M S$ ) $<2.7 \mathrm{~s}$.

We set the minimum detect cycle to 5 seconds, but it can be shortened to 3 seconds. 

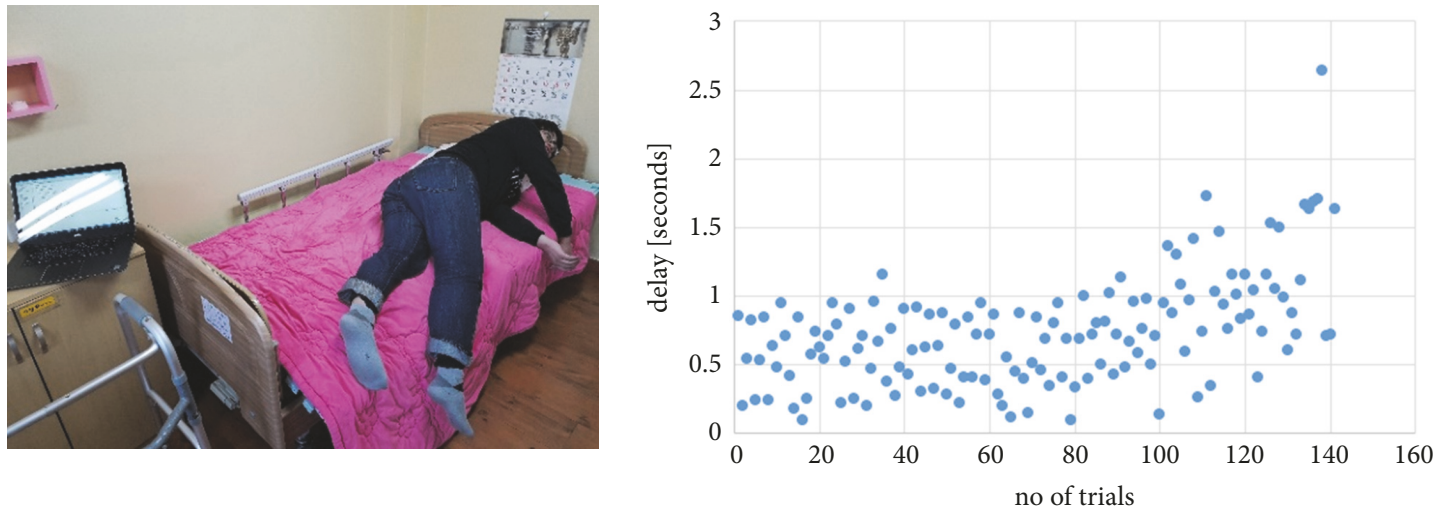

FIgURE 10: The experimental setup (left) and the distribution of the delays (right).

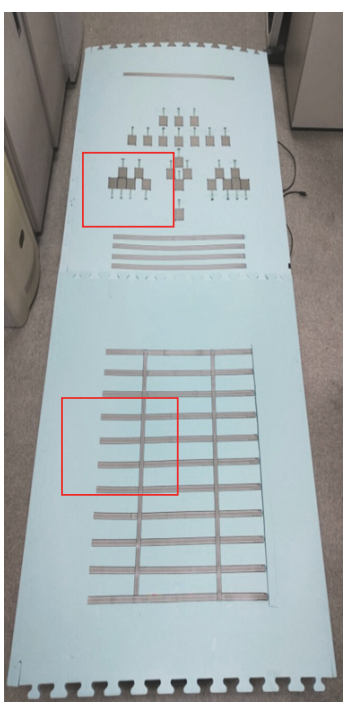

(a) The minimum D-zone

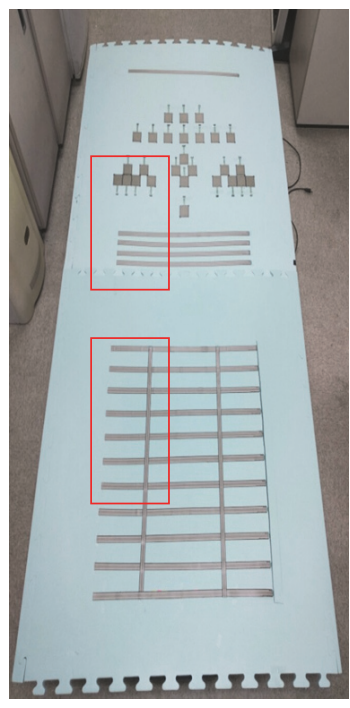

(b) The medium D-zone

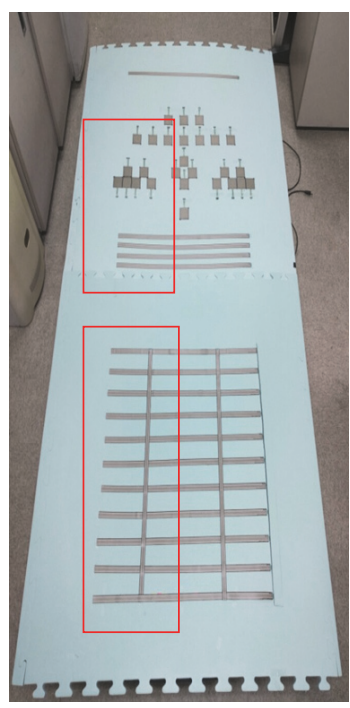

(c) The maximum D-zone

FIgURE 11: Analysis of detection time with respect to D-zone setting.

4.6. Detection of Bedsores. If a patient is the supine position, his (her) height can be estimated by using the distance between the occipital area sensors and the sensors that are deployed farthest from the occipital area sensors. Based on the average body size from the KATS, we know which sensors are sensed which position of a patient. It is important, because, according to KATS, bedsores frequently occur in specific locations.

In order to prevent bedsore attacks, caregivers should change the patient's posture at least every 2 hours [26]. Whenever the pressure of the specific position continues for longer than 60 minutes, the smart bed system gives caregivers warning messages. When the pressure continues for 90 minutes, it sends an alarm to the caregiver's smart device to change the patient's posture as soon as possible.

Figure 7 shows the duration time with respect to the patient posture. The green, orange, and red dots indicate the duration time of 30,60, and 90 minutes, respectively. As shown in Figure 12, our smart bed system can identify three types of patient posture: supine, lateral left, and lateral right. Figure 13 shows the patient's posture and accumulated pressure daily.

4.7. Guarantee of Comfort Sleep. The commercialized model is similar to the electric plate as shown in Figure 14. The controllers such as Raspberry PI boards will be placed on the side of the mattress. The survey results showed that more than $93 \%$ of users did not feel uncomfortable when lying down because of the smart mattress. To guarantee more comfort sleep, it is necessary to consider the material of smart mattress, which is beyond the scope of this paper.

\section{Conclusions}

Our smart bed prototype system can sense pressure occurring on the entire body of a patient with mobility impairment at a regular interval through an efficient deployment of sensors, while using a smaller number of sensors. In addition, the 


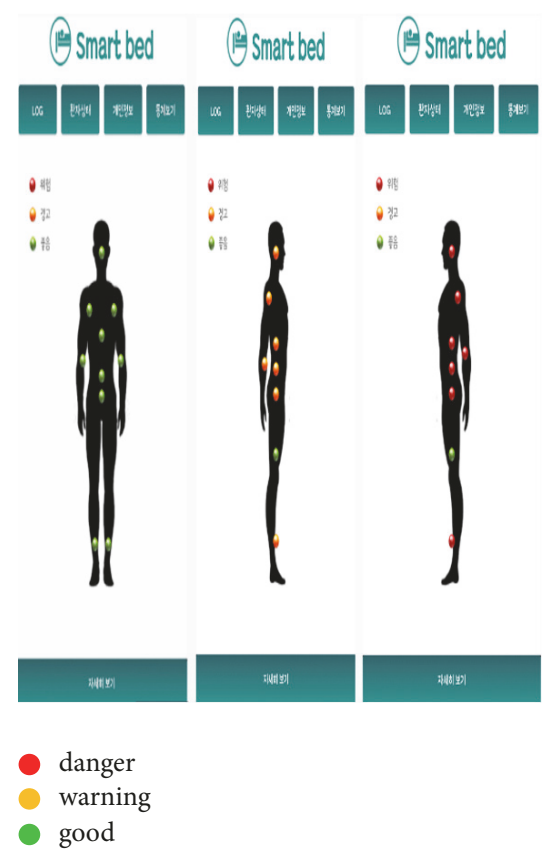

FIGURE 12: Visualization of the duration time of pressure with respect to the supine position (left) and the lateral position (center, right).
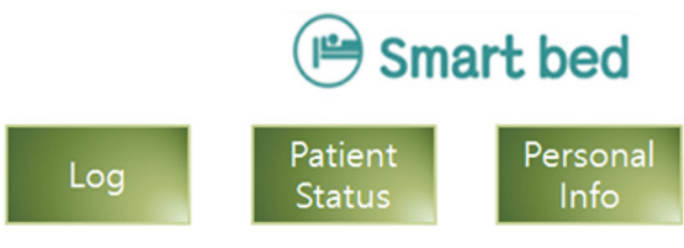

\section{Statistics}
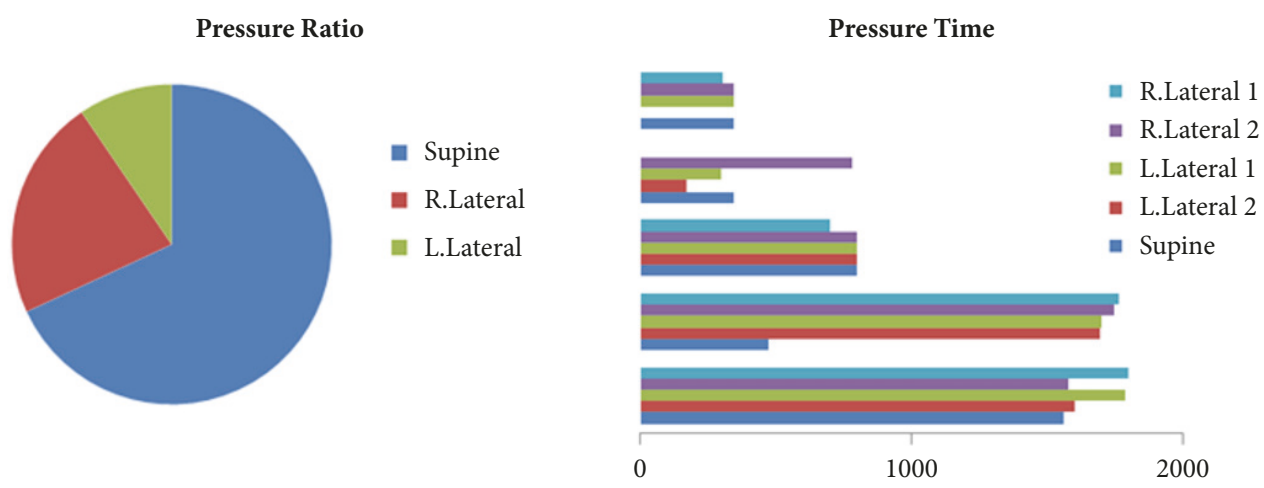

Supine : left heel, buttocks, left elbow, right wing bone, head Left lateral : heel, buttocks, spine, head

Right lateral : heel, buttocks, spine, head

FIGURE 13: Patient posture and accumulated pressure daily.

proposed system can detect the position with the highest possibility of bedsore attack by finding the largest accumulated pressure.

The proposed system can also provide the information about the position of the frequent occurrence of bedsore for caregivers depending on the patient's personal characteristics, for example, his (her) height or weight. Thus, it can help caregivers in terms of appropriately nursing their patients. Our system will be the one of the possible solutions to prevent bedsores and/or falling accidents and will be particularly useful in nursing homes and senior care centers.

At present, our smart bed can distinguish only three postures among elderly patients lying on the bed. If a patient is lying in a bent position, it may not be able to properly 


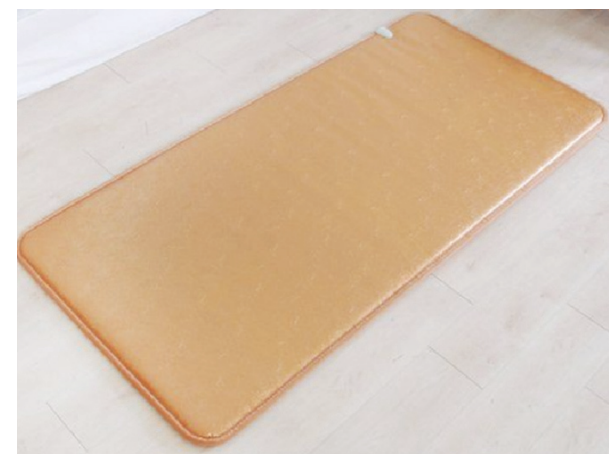

FIgURE 14: The prototype smart mattress (without controllers).

locate the pressure ulcer. Thus, we are currently studying ways to find various postures of patients more effectively. In the future, we will continue to investigate methods to measure the weight of elderly patients with impaired mobility even when they are lying down.

\section{Data Availability}

The data are generated at regular interval and stored in our local database system. Thus, all the data are stored in our local database system. These data will be given for proofing the correctness of our approach to the specific interests.

\section{Conflicts of Interest}

The author declares that there are no conflicts of interest regarding the publication of this paper.

\section{Acknowledgments}

This work is supported by the 2013 Grant of Incheon National University.

\section{References}

[1] "Nonstop Pressure," http://www.nursinghomeabuseguide.org/ neglect/bed-sores/.

[2] T. Yoshikawa, N. J. Livesley, and A. W. Chow, "Infected pressure ulcers in elderly individuals," Clinical Infectious Diseases, vol. 35, no. 11, pp. 1390-1396, 2002.

[3] M. Sartini, M. L. Cristina, A. M. Spagnolo et al., "The epidemiology of domestic injurious falls in a community dwelling elderly population: An outgrowing economic burden," European Journal of Public Health, vol. 20, no. 5, pp. 604-606, 2010.

[4] "Fall-Related Injuries," http://www.homecareassistancecincinnati.com/common-elderly-injuries/.

[5] G. D. Velhal, "Domestic accidents among the elderly," Annals of Tropical Medicine and Public Health, vol. 5, no. 2, pp. 61-62, 2012.

[6] Y.-O. Hwang, "Geriatric care facilities: recent trends in risk management," Journal of the Korean Gerontological Society, vol. 26, no. 3, pp. 505-520, 2006.

[7] S. M. R. Islam, D. Kwak, H. Kabir, M. Hossain, and K. Kwak, "The internet of things for health care: a comprehensive survey," IEEE Access, vol. 3, pp. 678-708, 2015.
[8] M. C. Domingo, "An overview of the internet of things for people with disabilities," Journal of Network and Computer Applications, vol. 35, no. 2, pp. 584-596, 2012.

[9] S. Amendola, R. Lodato, S. Manzari, C. Occhiuzzi, and G. Marrocco, "RFID technology for IoT-based personal healthcare in smart spaces," IEEE Internet of Things Journal, vol. 1, no. 2, pp. 144-152, 2014.

[10] M. Hassanalieragh, A. Page, T. Soyata et al., "Health monitoring and management using internet-of-things (IoT) sensing with cloud-based processing: opportunities and challenges," in Proceedings of the IEEE International Conference on Services Computing, SCC 2015, pp. 285-292, IEEE, July 2015.

[11] A. Pantelopoulos and N. G. Bourbakis, "A survey on wearable sensor-based systems for health monitoring and prognosis," IEEE Transactions on Systems, Man, and Cybernetics, Part C: Applications and Reviews, vol. 40, no. 1, pp. 1-12, 2010.

[12] W. Jung and Y. Hong, "A Design and Implementation of a Smart Bed for Elderly Patients," International Journal of Elderly Welfare Promotion and Management, vol. 1, no. 1, pp. 1-6, 2017.

[13] M. Yu, A. Rhuma, S. M. Naqvi, L. Wang, and J. Chambers, "A posture recognition-based fall detection system for monitoring an elderly person in a smart home environment," IEEE Transactions on Information Technology in Biomedicine, vol. 16, no. 6, pp. 1274-1286, 2012.

[14] Y. S. Delahoz and M. A. Labrador, "Survey on fall detection and fall prevention using wearable and external sensors," Sensors, vol. 14, no. 10, pp. 19806-19842, 2014.

[15] S. Gasparrini, E. Cippitelli, S. Spinsante, and E. Gambi, "A depth-based fall detection system using a kinect sensor," Sensors, vol. 14, no. 2, pp. 2756-2775, 2014.

[16] Kei-Myung University R\&DB Foundation, Fall-down prevent device, Patent No. 10-2013-0021614, 2014.8. 29 (registration date).

[17] Ó. D. Lara and M. A. Labrador, "A survey on human activity recognition using wearable sensors," IEEE Communications Surveys \& Tutorials, vol. 15, no. 3, pp. 1192-1209, 2013.

[18] K. Ozcan, A. K. Mahabalagiri, M. Casares, and S. Velipasalar, "Automatic fall detection and activity classification by a wearable embedded smart camera," IEEE Journal on Emerging and Selected Topics in Circuits and Systems, vol. 3, no. 2, pp. 125-136, 2013.

[19] HIMS Co. Ltd, System of unobtrusively recognizing actions and promoting physical activities and smart mat for the same purpose, Patent No. 10-2013-0024409, 2014. 4. 28 (registration date).

[20] "Smart Bed," http://boditrak.com/products/smartbeds.php.

[21] "Adaptive Sleep Thinking," http://www.elementsofrest.com/therest-bed/rest-technology.

[22] "Mattress Sensing MAP System," https://www.medgadget.com/ 2013/07/mattress-sensing-map-system-prevents-pressure-ulcers .html.

[23] "Symptoms of Pressure Ulcers", http://www.nhs.uk/Conditions/Pressure-ulcers/Pages/Symptoms.aspx.

[24] "Arduino Forum," http://forum.arduino.cc/index.php.

[25] “FSR-408 and FSR-406," https://cdn-shop.adafruit.com/datasheets/FSR400Series_PD.pdf.

[26] E.-S. Kwon, Study on the knowledge of bedsores care of a hospital nurse, recognition and execution, Hanyang University Graduate School of Public Administration, 2005. 


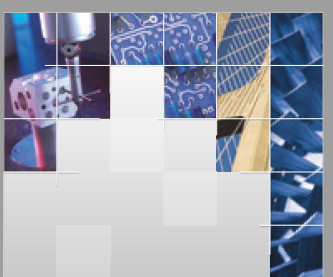

\section{Enfincering}
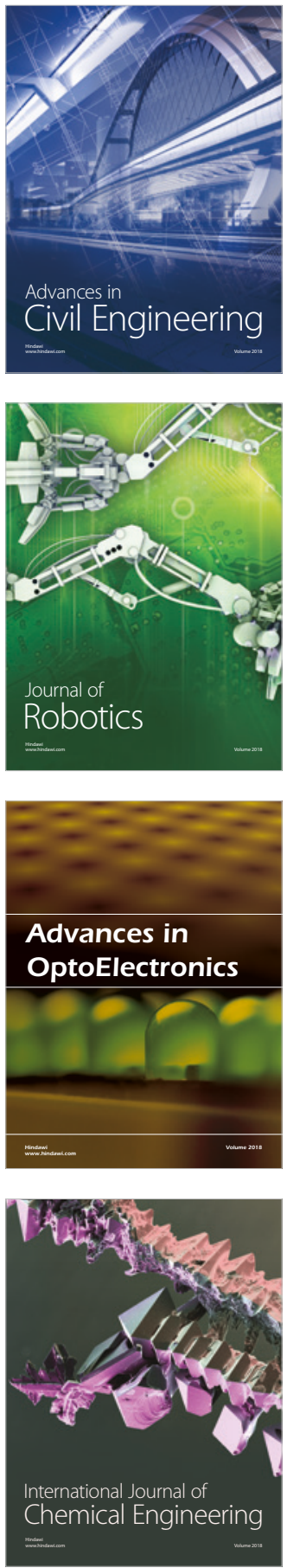

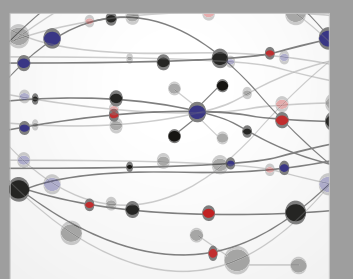

\section{Rotating \\ Machinery}

The Scientific World Journal

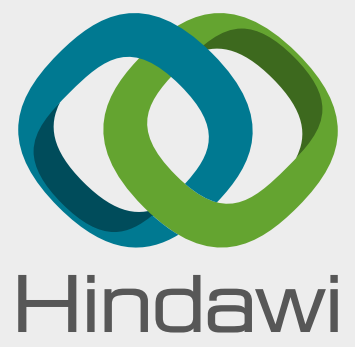

Submit your manuscripts at

www.hindawi.com
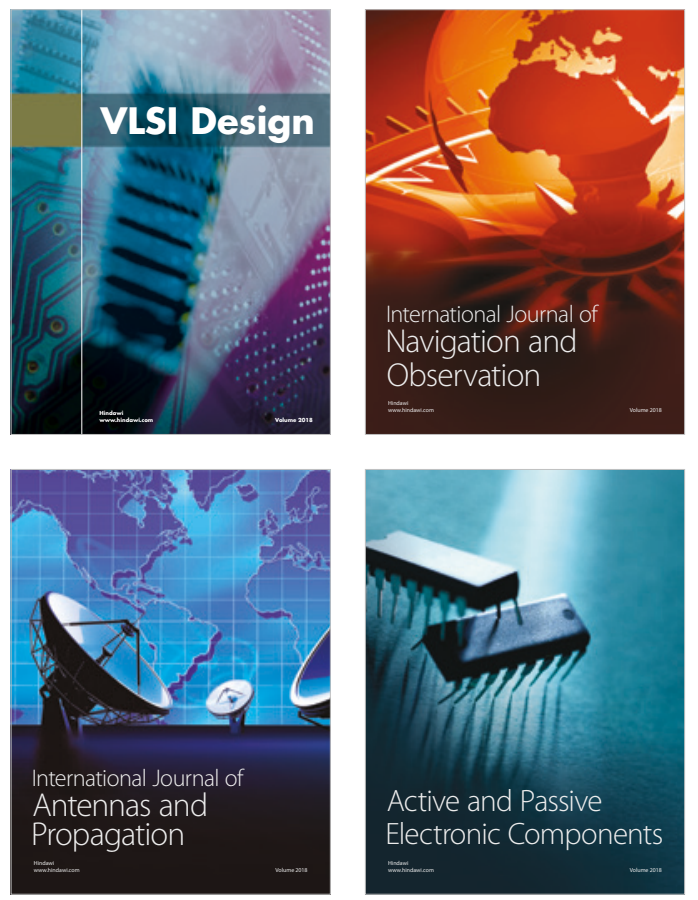
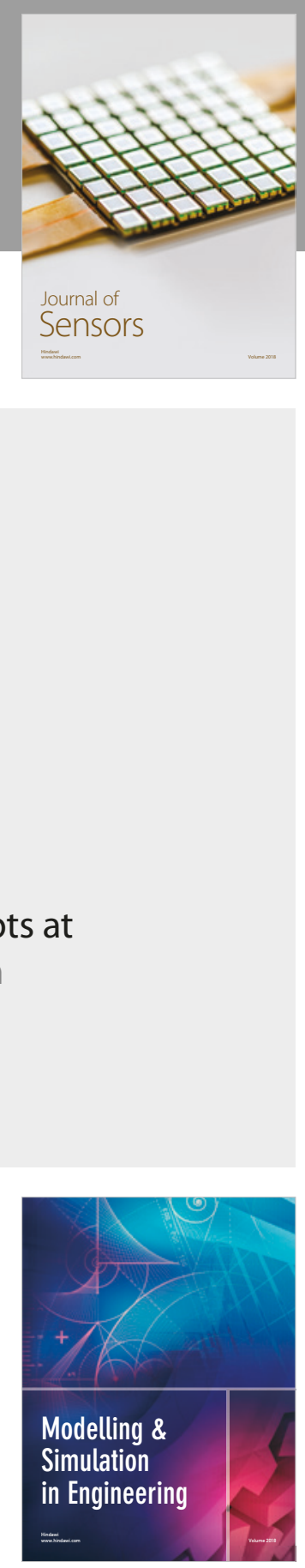

\section{Advances \\ Multimedia}
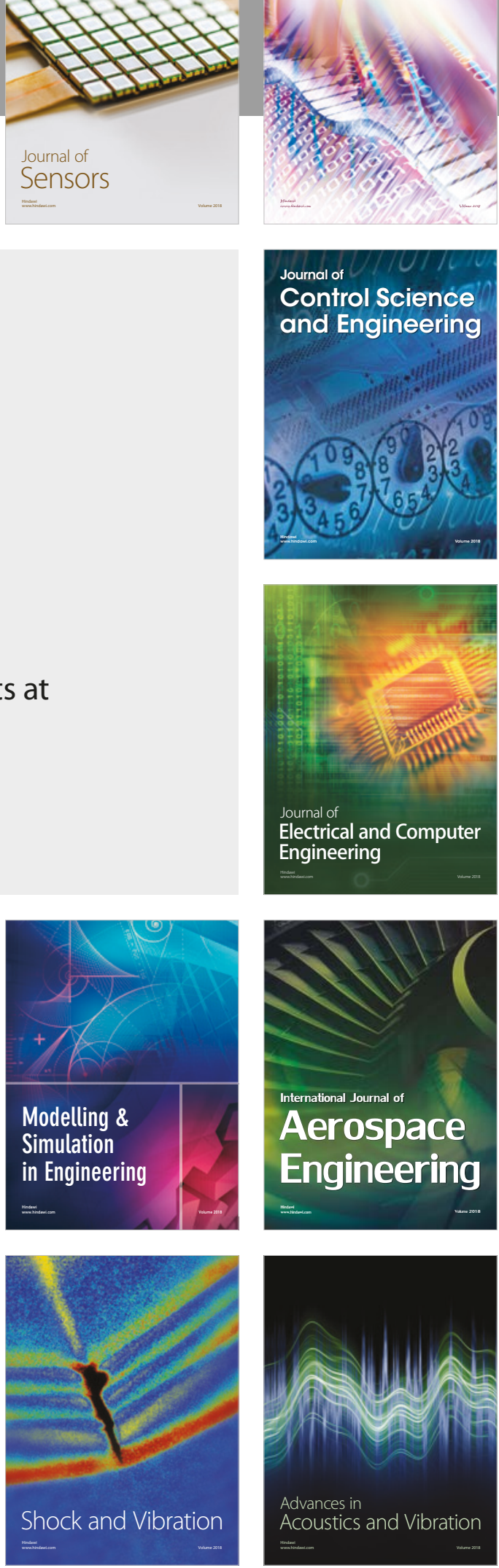